\title{
Exact and explicit approximate solutions to the multi-order fractional Burgers-Poisson and fractional Burgers-Poisson equations
}

\author{
Joshua Ikechukwu Nwamba \\ Department of Mathematics, University of Nigeria, Nsukka, 410001, Nigeria
}

Email address:

jnwam2day@yahoo.com

To cite this article:

Joshua Ikechukwu Nwamba. Exact and Explicit Approximate Solutions to the Multi-Order Fractional Burgers-Poisson and Fractional Burgers-Poisson Equations. Applied and Computational Mathematics. Vol. 2, No. 3, 2013, pp. 78-85. doi: 10.11648/j.acm.20130203.12

\begin{abstract}
The multi-order fractional Burgers-Poisson (MFBP) equation was introduced, exact as well as approximate solutions to the introduced MFBP, fractional Burgers-Poisson (fBP) and Burgers-Poisson (BP) equations were obtained through the use of the homotopy perturbation method (HPM) and the Adomian decomposition method (ADM) in this paper. The effectiveness and efficiency of the approximate techniques in handling strongly nonlinear multi-order fractional as well as fractional partial differential equations was established in this paper. It was also shown in this paper that the two approximate techniques employed gave similar results to the considered model equations.
\end{abstract}

Keywords: Homotopy Perturbation Method, Adomian Decomposition Method, Burgers-Poisson Equation, Lie Method, Dispersive Media, Multi-Order Fractional Differential Equations

\section{Introduction}

It was observed in [1] that (2) properly describe the unidirectional propagation of long waves in dispersive media. For unidirectional water waves exhibiting weaker dispersive effects, the BP equation (a shallow water equation) turned out to be a better model equation compared to the Korteg-de-Vires (KDV) equation. Due to this, its importance in the area of mathematical physics and continuum mechanics cannot be overemphasized.

Notable among the interesting behaviors exhibited by the BP equation were, local existence results for smooth solutions, global existence result for weak entropy solutions and wave breaking in finite time [1]. The classical Lie method was employed in constructing group invariant solutions to (2) in [2], while [3] used the variational iteration method to obtain an accurate approximate solution to the BP equation. Employing Pade $(2,2)$ approximation, [4] proposed that the BP equation gives the phase velocity which arises in linear water wave theory. In [5], large time behavior of global solutions to (2) was considered.
Where $u(x, t)$ denotes the velocity of a given gas and $\varphi(x, t)$, the radiative heat-flux, the system (2) with the initial condition $u(x, 0)=u_{0}(x)$ can be used to describe the behavior of a high temperature gas $[6,7]$.

The multi-order fractional Burgers-Poisson equation can be represented as,

$$
\begin{aligned}
& D_{t}^{\alpha} u(x, t)-D_{t}^{\alpha} D_{x}^{\beta} u(x, t)+D_{x}^{\alpha^{\prime}} u(x, t)(1+u(x, t)) \\
& =3 D_{x}^{\alpha^{\prime}} u(x, t) D_{x}^{\beta} u(x, t)+u(x, t) D_{x}^{\delta} u(x, t) \\
& 0<\alpha, \alpha^{\prime} \leq 1,1<\beta \leq 2,2<\delta \leq 3, t>0
\end{aligned}
$$

The classical Burgers-Poisson (BP) equation is given as

$$
\begin{aligned}
& u_{t}+u u_{x}+\varphi_{x}=0, \\
& -\varphi_{x x}+\varphi+u=0
\end{aligned}
$$

We may multiply both sides of (2a) with $\left(1-\partial_{x}^{2}\right)$ and employing ( $2 b)$ on the resulting right hand side (r.h.s), we obtain,

$$
u_{t}-u_{x x t}+u_{x}+u u_{x}-3 u_{x} u_{x x}-u u_{x x x}=0
$$


Furthermore, where $\varphi_{x}<1,0<x, t<\infty$ in (2), we may neglect $\varphi_{x x}$ to formally obtain

$$
\begin{aligned}
& u_{t}+u u_{x}+\varphi_{x}=0 \\
& \varphi=-u
\end{aligned}
$$

The resulting implication of the above condition is that $u_{x x}$ may be neglected in (3) to also obtain (4) since $u_{x}$ is very small.

Also, the fractional BP equation is represented as

$$
\begin{aligned}
& D_{t}^{\alpha} u(x, t)-D_{t}^{\alpha} u_{x}(x, t)+u_{x}(x, t)(1+u(x, t)) \\
& =3 u_{x}(x, t) u_{x x}(x, t)+u(x, t) u_{x x x}(x, t) \\
& 0<\alpha \leq 1, t>0
\end{aligned}
$$

where $\varphi$ and $u$ depend on $(t, x) \in(0, \infty) \times R$, and subscripts denote partial derivatives, $\alpha, \alpha^{\prime}, \beta$ and $\delta$ denotes the fractional time and space derivatives in the Caputo's sense. The function $u(x, t)$ becomes zero for all $x, t<0$ as $x, t \rightarrow-\infty$.

In the case where $\delta=3, \beta=2$ and $\alpha, \alpha^{\prime}=1$, the multiorder fractional equation (1) reduces to the classical Burger's-Poisson (BP) equation given by (2). Furthermore, in the case $\delta=3, \beta=2$ and $\alpha^{\prime}=1$, we obtain the fractional $\mathrm{BP}$ equation given by (5) and treated in [8].

In this paper, the homotpy perturbation method (HPM) [9-13] and Adomian decomposition method (ADM) [14-18] will be employed to obtain both exact and approximate explicit solutions of the multi-order fractional Burger'sPoisson (MFBP) equation and the time-fractional BurgersPoisson (fBP) equation.

\section{Essential Preliminaries}

\subsection{Fractional Calculus}

In recent times, it is obvious that the development and application of fractional calculus in many fields has yielded substantial results. As a result, areas where fractional calculus has been applied spans, acoustics, electromagnetic, electrochemistry, viscoelasticity, material science, to mention only but a few. The importance of fractional calculus stems from the fact that, for physical phenomena depending not only on the present time but the past time history, it has proved effective and efficient.(see $[8,19])$ Moreover, multi-order fractional differential equations play huge roles in the fields mentioned above, popular ones include, the Bagley-Torvick equation [20-22] and the Basset equation [22,23]. Very few literatures (see [22, 24]) exist where efforts have been made to solve multi-order fractional differential equations, yet a thorough and comprehensive analysis has not surfaced.

First, we define the concept of fractional-order integration and fractional-order differentiation [25]. In defining the concept of fractional derivative we will adopt Caputo's definition whose advantage over the RiemannLiouville definition lies in its ability to handle Initial Value Problems (IVPs) properly.

Definition 2.10: A real function $f(t)$ is said to be in the space $S_{\mu}, \mu \in R$, if there exists a real number $n>\mu$, such that $t^{-p} f(t)=f_{1}(t)$, where $f_{1}(t) \in S(0, \infty)$ and it is said to be in the space $S_{p}$ if and only if $f^{p} \in S_{\mu}, p \in N$.

Definition 2.11: The Riemann-Liouville fractional integral operator of order $\alpha, I^{\alpha}$ of a function $f \in S_{\mu}, \mu \geq-1$ is defined as

$$
I^{\alpha} f(t)=\frac{1}{\Gamma(\alpha)} \int_{0}^{t}(t-\tau)^{\alpha-1} f(\tau) d \tau, t>0, \alpha>0
$$

Definition 2.12: The fractional derivative in Caputo's sense is defined as

$$
D^{\alpha} f(t)=\frac{1}{\Gamma(m-\alpha)} \int_{0}^{t}(t-\tau)^{m-1-\alpha} f^{(m)}(\tau) d \tau
$$

where $\alpha$ is the order of operation, $m$ satisfies the relation $m-1<\alpha \leq m, m \in N, t>0, f \in S_{-1}^{m}$.

Definition 2.13: The Caputo time-fractional derivative operator of order $\alpha>0$ will be defined as

$$
D^{\alpha} u(x, t)=\left\{\begin{array}{c}
\frac{1}{\Gamma(m-\alpha)} \int_{0}^{t} \frac{\partial^{m} u(x, \tau)}{\partial \tau^{m}}(t-\tau)^{m-1-\alpha} d \tau, \text { if } m-1<\alpha>0 \leq m \\
\frac{\partial^{m} u(x, t)}{\partial t^{m}}, \text { if } \alpha=m \in N
\end{array}\right.
$$

if $m$ were to be the smallest integer that exceeds $\alpha$.

Lemma 2.1: In the case where $m-1<\alpha>0 \leq m, m \in N$ and $f \in S_{\mu}, \mu \geq-1$, then

$$
I^{\alpha} D^{\alpha} f(t)=f(t)-\sum_{k=0}^{m-1} f\left(0^{+}\right) \frac{t^{k}}{k !}
$$

We may set $\alpha=n \alpha, n \geq 1$ in (6) and (7) to obtain the useful relations

$$
\begin{aligned}
& I^{n \alpha} f(t)=\frac{1}{\Gamma(n \alpha)} \int_{0}^{t}(t-\tau)^{n \alpha-1} f(\tau) d \tau, t>0, n \alpha>0 \\
& D^{\alpha} f(t)=\frac{1}{\Gamma(m-n \alpha)} \int_{0}^{t}(t-\tau)^{m-1-n \alpha} f^{(m)}(\tau) d \tau
\end{aligned}
$$

Furthermore, (10) and (11) can be respectively rewritten for $f(t)=t^{k}$ as

$$
I^{n \alpha} t^{k}=\frac{\Gamma(k+1) t^{k+n \alpha}}{\Gamma(k+n \alpha+1)}
$$




$$
D^{n \alpha} t^{k}=\frac{\Gamma(k+1) t^{k-n \alpha}}{\Gamma(k-n \alpha+1)}
$$

For comprehensive and heuristic study, see[25-30].

\subsection{Homotopy Perturbation Method (HPM)}

This powerful semi-analytic method of obtaining approximate solutions to varying Differential equations (D.E's) was first proposed by Him in [9]. Over the years, it has been reviewed and improved to increase the convergence rate and accommodate modern challenges [10-13].

Consider the following general nonlinear differential equation of the form

$$
A(u)-f(r)=0, r \in \Omega
$$

with the corresponding boundary conditions

$$
B\left(u, \frac{\partial u}{\partial n}\right)=0, r \in \Gamma
$$

where $A$ is a general differential operator, $B$ is the boundary operator, $f(r)$ is a known analytic function and $\Gamma$ is the boundary of the domain $\Omega$.

The operator $A$ can be generally divided into linear $(L)$ and nonlinear $(N)$ parts. Then, we may write (14) as follows:

$$
L(u)+N(u)-f(r)=0
$$

Employing the Homotopy technique, we construct a homotopy $U(r, p): \Omega \times[0,1] \rightarrow R$, which satisfies

$$
\begin{aligned}
& H(U, p)=(1-p)\left[L(U)-L\left(U_{0}\right)\right] \\
& +p[A(U)-f(r)]=0, p \in[0,1], r \in \Omega
\end{aligned}
$$

or

$$
\begin{aligned}
& H(U, p)=L(U)-L\left(U_{0}\right)+p L\left(U_{0}\right) \\
& +p[N(U)-f(r)]=0
\end{aligned}
$$

where $p \in[0,1]$, is an embedding parameter, $U_{0}$ is an initial approximation for the solution of (14) which must satisfy the given boundary conditions in (15). Obviously, from (17) and (18) we have

$$
\begin{gathered}
H(U, 0)=L(U)-L\left(U_{0}\right)=0 \\
H(U, 1)=A(U)-f(r)=0
\end{gathered}
$$

The fact that as $p$ changes from zero to unity, $U(r, p)$ varies from $U_{0}$ to $u$ is termed homotopy (continuous deformation) in topology. On the account of the HPM, we can assume that the solution of (17) and (18) can be written as a power series in $p$ :

$$
U=U_{0}+p U_{1}+p^{2} U_{2}+\ldots
$$

Then, letting $p=1$, we obtain the solution of (14)

$$
u=\lim _{p \rightarrow 1} U=U_{0}+U_{1}+U_{2}+\ldots
$$

It has been proved that the series (22) converges [11].

\subsection{Adomian Decomposition Method (ADM)}

This method first proposed by G. Adomian [14], has proved efficient and effective in handling nonlinear D.E problems of Engineering, Applied Mathematics and Physics. (see [15-19])

Consider again (14) in the form of (16) for $0 \leq t \leq 1$ where $L=\frac{d^{n}}{d t^{n}}$ is the $n$-order derivative operator. We assume that the inverse linear operator $L^{-1}$ exists and can be conveniently taken as the definite integral for a function in the following form

$$
L^{-1}(.)=\int_{0}^{x} \int_{0}^{t_{1}} \int_{0}^{t_{n-1}} \ldots \int_{0}^{t_{n}}(.) d t_{1} \ldots d t_{n-1} d t_{n}
$$

Applying the inverse operator to both sides of (16) yields

$$
L^{-1} L(u)=L^{-1} f-L^{-1} N(u)
$$

Thus, we have

$$
u(t)=\sum_{k=0}^{n-1} \frac{t^{k}}{k !} u^{k}(0)-L^{-1} N(u)+L^{-1} f
$$

Assume for $0 \leq k \leq n-1$,

$$
A_{k}=u^{k}(0)=\left.\frac{1}{k !} \frac{d^{k}}{d \lambda^{k}}\left[N\left[\sum_{n=0}^{\infty} \lambda^{n} u_{n}\right]\right]\right|_{\lambda=0}, n \geq 1
$$

Equation (26) gives the so called Adomian polynomials .

Then according to the ADM, the solution of (16) is given as

$$
u(t)=\sum_{k=0}^{\infty} u_{n}(t)
$$

with $u_{0}(t)=u(0)$.

\section{Application of HPM}

Consider (1) with the initial conditions 


$$
u(x, 0)=x
$$

The exact solution of the BP equation, a special case of MFBP when $\alpha=\alpha^{\prime}=1, \beta=2, \delta=3$, is given by classical Lie method (see [2]) as

$$
u(x, t)=\frac{1+x}{1+t}-1
$$

According to the HPM, the homotopy is constructed as follows:

$$
(1-p) D_{t}^{\alpha} U+p\left[D_{t}^{\alpha} U-D_{t}^{\alpha} D_{x}^{\beta} U+D_{x}^{\alpha^{\prime}} U(1+U)-\left(3 D_{x}^{\alpha^{\prime}} U D_{x}^{\beta} U+U D_{x}^{\delta} U\right)\right]=0
$$

or

$$
D_{t}^{\alpha} U+p\left[-D_{t}^{\alpha} D_{x}^{\beta} U+D_{x}^{\alpha^{\prime}} U(1+U)-\left(3 D_{x}^{\alpha^{\prime}} U D_{x}^{\beta} U+U D_{x}^{\delta} U\right)\right]=0
$$

where $p \in[0,1]$ is an embedding parameter. By utilizing the parameter $p$, the solution $u(x, t)$ can be expanded in the following form

$$
u(x, t)=U_{0}(x, t)+p U_{1}(x, t)+p^{2} U_{2}(x, t)+p^{3} U_{3}(x, t)+\ldots
$$

Setting $p=1$ gives the approximate solution

$$
u(x, t)=U_{0}(x, t)+U_{1}(x, t)+U_{2}(x, t)+U_{3}(x, t)+\ldots
$$

Now substituting (32) into (31) and equating the terms with the identical powers of $p$ gives

$$
\begin{aligned}
& p^{0}: D_{t}^{\alpha} U_{0}=0, \quad U_{0}(x, 0)=x ; \\
& p^{1}: D_{t}^{\alpha} U_{1}-D_{t}^{\alpha} D_{x}^{\beta} U_{0}+D_{x}^{\alpha^{\prime}} U_{0}\left(1+U_{0}\right) \\
& -3 D_{x}^{\alpha^{\prime}} U_{0} D_{x}^{\beta} U_{0}-U_{0} D_{x}^{\delta} U_{0}=0, U_{1}(x, 0)=0 ; \\
& p^{2}: D_{t}^{\alpha} U_{2}-D_{t}^{\alpha} D_{x}^{\beta} U_{1}+D_{x}^{\alpha^{\prime}} U_{1}\left(1+U_{0}\right) \\
& +U_{1} D_{x}^{\alpha^{\prime}} U_{0}-3\left(D_{x}^{\alpha^{\prime}} U_{0} D_{x}^{\beta} U_{1}+D_{x}^{\alpha^{\prime}} U_{1} D_{x}^{\beta} U_{0}\right) \\
& -\left(U_{0} D_{x}^{\delta} U_{1}+U_{1} D_{x}^{\delta} U_{0}\right)=0, U_{2}(x, 0)=0 \\
& p^{3}: D_{t}^{\alpha} U_{3}-D_{t}^{\alpha} D_{x}^{\beta} U_{2}+D_{x}^{\alpha^{\prime}} U_{2} \\
& +\left(U_{0} D_{x}^{\alpha^{\prime}} U_{2}+U_{1} D_{x}^{\alpha^{\prime}} U_{1}+U_{2} D_{x}^{\alpha^{\prime}} U_{0}\right) \\
& -3\left(D_{x}^{\alpha^{\prime}} U_{0} D_{x}^{\beta} U_{2}+D_{x}^{\alpha^{\prime}} U_{1} D_{x}^{\beta} U_{1}+D_{x}^{\alpha^{\prime}} U_{2} D_{x}^{\beta} U_{0}\right) \\
& -\left(U_{0} D_{x}^{\delta} U_{2}+U_{1} D_{x}^{\delta} U_{1}+U_{2} D_{x}^{\delta} U_{0}\right)=0, \\
& U_{3}(x, 0)=0 \\
& p^{n}: D_{t}^{\alpha} U_{n}=D_{t}^{\alpha} D_{x}^{\beta} U_{n-1}-D_{x}^{\alpha^{\prime}} U_{n-1} \\
& -\sum_{m=0}^{n-1} U_{m} D_{x}^{\alpha^{\prime}} U_{n-1-m}+3 \sum_{m=0}^{n-1} D_{x}^{\alpha^{\prime}} U_{m} D_{x}^{\beta} U_{n-1-m}
\end{aligned}
$$

$$
+\sum_{m=0}^{n-1} U_{m} D_{x}^{\delta} U_{n-1-m}, \quad U_{n}(x, 0)=0, n \geq 1
$$

Applying the operator (10) to (34) and utilizing lemma 2.1, (12) and (13), we obtain the approximate solution to the MFBP equation given as

$$
U_{n}=I_{t}^{\alpha}\left[D_{t}^{\alpha} D_{x}^{\beta} U_{n-1}-D_{x}^{\alpha^{\alpha}} U_{n-1}-\sum_{m=0}^{n-1} U_{m} D_{x}^{\alpha^{\prime}} U_{n-1-m}+3 \sum_{m=0}^{n-1} D_{x}^{\alpha^{\alpha}} U_{m} D_{x}^{\beta} U_{n-1-m}+\sum_{m=0}^{n-1} U_{m} D_{x}^{\delta} U_{n-1-m}\right]
$$

Thus,

$$
\begin{gathered}
U_{0}(x, t)=x \\
U_{1}(x, t)=-\frac{x^{1-\alpha^{\prime}}(1+x)}{\Gamma(1+\alpha) \Gamma\left(2-\alpha^{\prime}\right)} t^{\alpha} \\
U_{2}(x, t)=\frac{\alpha^{\prime}\left(1-\alpha^{\prime}\right) x^{1-\alpha^{\prime}-\beta}}{\Gamma(1+\alpha) \Gamma\left(2-\alpha^{\prime}\right) \Gamma(3-\beta)} t^{\alpha}+\frac{x^{1-2 \alpha^{\prime}}}{\Gamma(1+2 \alpha) \Gamma\left(2-\alpha^{\prime}\right)} t^{2 \alpha} \\
\left.\frac{\Gamma\left(2-\alpha^{\prime}\right)}{\Gamma\left(2-2 \alpha^{\prime}\right)}+x\left(\frac{\Gamma\left(2-\alpha^{\prime}\right)}{\Gamma\left(2-2 \alpha^{\prime}\right)}+\frac{\Gamma\left(3-\alpha^{\prime}\right)}{\Gamma\left(3-2 \alpha^{\prime}\right)}\right)+\frac{x^{2} \Gamma\left(3-\alpha^{\prime}\right)}{\Gamma\left(3-2 \alpha^{\prime}\right)}\right] \\
+\frac{x^{2-2 \alpha^{\prime}}(1+x)}{\Gamma(1+2 \alpha) \Gamma\left(2-\alpha^{\prime}\right)^{2}} t^{2 \alpha}+\frac{3 \alpha^{\prime}\left(1-\alpha^{\prime}\right) x^{2-2 \alpha^{\prime}-\beta}}{\Gamma(1+2 \alpha) \Gamma\left(2-\alpha^{\prime}\right) \Gamma(3-\beta)} t^{2 \alpha} \\
-\frac{\alpha^{\prime}\left(1-\alpha^{\prime}\right)\left(1+\alpha^{\prime}\right) x^{2-\alpha^{\prime}-\delta}}{\Gamma(1+2 \alpha) \Gamma\left(2-\alpha^{\prime}\right) \Gamma(4-\beta)} t^{2 \alpha}
\end{gathered}
$$

where the first four approximations of (33) are sufficient, then the approximate explicit solution of (1) with the initial condition (28) will be expressed as the combination of (36)-(39).

Table 1.

\begin{tabular}{ccccc}
\hline $\mathbf{t}$ & Exact & HPM\&ADM & Error & Percentage Error \\
\hline 0.0000 & 2.0000 & 2.0000 & 0.0000 & 0.0000 \\
0.2000 & 1.4000 & 1.3994 & 0.0006 & 0.4280 \\
0.4000 & 1.0000 & 0.9688 & 0.0312 & 3.1200 \\
0.6000 & 0.7143 & 0.4092 & 0.3051 & 42.710 \\
0.8000 & 0.5000 & -1.0000 & 1.5000 & 300.00
\end{tabular}

Table 2.

\begin{tabular}{ccccc}
\hline $\mathbf{t}$ & Exact & HPM\&ADM & Error & Percentage Error \\
\hline 0.0000 & -0.1667 & -0.1680 & 0.0013 & -0.7798 \\
0.5000 & 0.2963 & 0.2942 & 0.0021 & 0.7087 \\
1.0000 & 0.7593 & 0.7564 & 0.0029 & 0.3819 \\
1.5000 & 1.2222 & 1.2187 & 0.0035 & 0.2864 \\
2.0000 & 1.6852 & 1.6809 & 0.0043 & 0.2552 \\
2.5000 & 2.1481 & 2.1431 & 0.0050 & 0.2328
\end{tabular}




$\begin{array}{lllll}3.0000 & 2.6111 & 2.6053 & 0.0058 & 0.2221 \\ 3.5000 & 3.0741 & 3.0676 & 0.0065 & 0.2114 \\ 4.0000 & 3.5370 & 3.5300 & 0.0070 & 0.1979 \\ 4.5000 & 4.0000 & 3.9920 & 0.0080 & 0.2000\end{array}$

$$
\begin{aligned}
& U_{3}(x, t)=t^{\alpha} \frac{\alpha^{\prime}\left(1-\alpha^{\prime}\right) \Gamma\left(2-\alpha^{\prime}-\beta\right) x^{1-\alpha^{\prime}-2 \beta}}{\Gamma(1+\alpha) \Gamma\left(2-\alpha^{\prime}\right) \Gamma(3-\beta) \Gamma\left(2-\alpha^{\prime}-2 \beta\right)} \\
& +\frac{x^{1-2 \alpha^{\prime}-\beta}}{\Gamma(1+2 \alpha)} t^{2 \alpha}\left[\begin{array}{c}
\frac{1}{\Gamma\left(2-2 \alpha^{\prime}-\beta\right)}+x\left(\frac{4-3 \alpha^{\prime}}{\Gamma\left(3-2 \alpha^{\prime}-\beta\right)}+\frac{\Gamma\left(3-2 \alpha^{\prime}\right)}{\Gamma\left(2-\alpha^{\prime}\right)^{2} \Gamma\left(3-2 \alpha^{\prime}-\beta\right)}\right)+x^{2}\left(\frac{\left(3-2 \alpha^{\prime}\right)\left(2-\alpha^{\prime}\right)}{\Gamma\left(4-2 \alpha^{\prime}-\beta\right)}+\frac{\Gamma\left(4-2 \alpha^{\prime}\right)}{\Gamma\left(2-\alpha^{\prime}\right)^{2} \Gamma\left(4-2 \alpha^{\prime}-\beta\right)}\right) \\
+\frac{3 \alpha^{\prime}\left(1-\alpha^{\prime}\right) \Gamma\left(3-2 \alpha^{\prime}-\beta\right) x^{1-\beta}}{\Gamma\left(2-\alpha^{\prime}\right)^{2} \Gamma(3-\beta) \Gamma\left(3-2 \alpha^{\prime}-2 \beta\right)}-\frac{\alpha^{\prime}\left(1+\alpha^{\prime}\right) \Gamma\left(3-\alpha^{\prime}-\delta\right) x^{1+\alpha^{\prime}-\delta}}{\Gamma\left(1-\alpha^{\prime}\right) \Gamma(4-\delta) \Gamma\left(3-\alpha^{\prime}-\beta-\delta\right)}-\left[\begin{array}{c}
\frac{\alpha^{\prime}\left(1+\alpha^{\prime}\right) \Gamma\left(2-\alpha^{\prime}-\beta\right)}{\Gamma\left(1-\alpha^{\prime}\right) \Gamma(3-\beta) \Gamma\left(2-2 \alpha^{\prime}-\beta\right)} \\
+\frac{\alpha \Gamma\left(2-\alpha^{\prime}-\beta\right) x}{\Gamma\left(1-\alpha^{\prime}\right) \Gamma(3-\beta) \Gamma\left(2-2 \alpha^{\prime}-\beta\right)}
\end{array}\right] \\
\frac{\alpha \Gamma\left(2-\alpha^{\prime}-\beta\right) x^{1-\alpha^{\prime}}}{\Gamma\left(1-\alpha^{\prime}\right) \Gamma(3-\beta) \Gamma\left(2-2 \alpha^{\prime}-\beta\right) \Gamma\left(2-\alpha^{\prime}\right)}+\frac{3 \alpha \Gamma\left(2-\alpha^{\prime}-\beta\right) x^{1-\beta}}{\Gamma\left(2-\alpha^{\prime}\right) \Gamma\left(1-\alpha^{\prime}\right) \Gamma(3-\beta) \Gamma\left(2-\alpha^{\prime}-2 \beta\right)}-\frac{\alpha \Gamma\left(3-\alpha^{\prime}-\delta\right) x^{1+\alpha^{\prime}-\delta}}{\Gamma\left(1-\alpha^{\prime}\right) \Gamma(3-\beta) \Gamma\left(2-\alpha^{\prime}-\beta-\delta\right)}
\end{array}\right] \\
& {\left[(1+x)\left(\begin{array}{l}
\frac{1}{\Gamma\left(2-3 \alpha^{\prime}\right)}+x\left(\frac{\left(4-3 \alpha^{\prime}\right)}{\Gamma\left(3-3 \alpha^{\prime}\right)}+\frac{\Gamma\left(3-2 \alpha^{\prime}\right)}{\Gamma\left(2-\alpha^{\prime}\right)^{2} \Gamma\left(3-3 \alpha^{\prime}\right)}\right)+x^{2}\left(\frac{\left(3-2 \alpha^{\prime}\right)\left(2-\alpha^{\prime}\right)}{\Gamma\left(4-3 \alpha^{\prime}\right)}+\frac{\Gamma\left(4-2 \alpha^{\prime}\right)}{\Gamma\left(2-\alpha^{\prime}\right)^{2} \Gamma\left(4-3 \alpha^{\prime}\right)}\right) \\
\frac{3 \alpha^{\prime}\left(1-\alpha^{\prime}\right) \Gamma\left(3-2 \alpha^{\prime}-\beta\right) x^{1-\beta}}{\Gamma\left(2-\alpha^{\prime}\right)^{2} \Gamma(3-\beta) \Gamma\left(3-3 \alpha^{\prime}-\beta\right)}-\frac{\alpha^{\prime}\left(1+\alpha^{\prime}\right) \Gamma\left(3-\alpha^{\prime}-\delta\right) x^{1+\alpha^{\prime}-\delta}}{\Gamma\left(1-\alpha^{\prime}\right) \Gamma(4-\delta) \Gamma\left(3-2 \alpha^{\prime}-\delta\right)}
\end{array}\right)\right.} \\
& -\frac{x}{\Gamma\left(2-3 \alpha^{\prime}\right)}-\left(\frac{\left(4-3 \alpha^{\prime}\right)}{\Gamma\left(3-3 \alpha^{\prime}\right)}+\frac{\Gamma\left(3-2 \alpha^{\prime}\right)}{\Gamma\left(2-\alpha^{\prime}\right)^{2} \Gamma\left(3-3 \alpha^{\prime}\right)}\right) x^{2}-\left(\frac{\left(2-\alpha^{\prime}\right)\left(3-2 \alpha^{\prime}\right)}{\Gamma\left(4-3 \alpha^{\prime}\right)}+\frac{\Gamma\left(4-2 \alpha^{\prime}\right)}{\Gamma\left(2-\alpha^{\prime}\right)^{2} \Gamma\left(4-3 \alpha^{\prime}\right)}\right) x^{3} \\
& \frac{3 \alpha \Gamma\left(3-2 \alpha^{\prime}-\beta\right) x^{2-\beta}}{\Gamma\left(1-\alpha^{\prime}\right) \Gamma\left(2-\alpha^{\prime}\right)^{2} \Gamma(3-\beta) \Gamma\left(3-3 \alpha^{\prime}-\beta\right)}+\frac{\alpha^{\prime}\left(1+\alpha^{\prime}\right) \Gamma\left(3-\alpha^{\prime}-\delta\right) x^{2+\alpha-\delta}}{\Gamma\left(1-\alpha^{\prime}\right) \Gamma(4-\delta) \Gamma\left(3-2 \alpha^{\prime}-\delta\right)}-\frac{(1+x)(\Gamma(1+2 \alpha))}{\Gamma(1+\alpha)^{2} \Gamma\left(2-\alpha^{\prime}\right)} \\
& \left(\frac{\Gamma\left(2-\alpha^{\prime}\right) x}{\Gamma\left(2-2 \alpha^{\prime}\right)}+\frac{\Gamma\left(3-\alpha^{\prime}\right) x^{2}}{\Gamma\left(3-2 \alpha^{\prime}\right)}\right)-\frac{x^{1-\alpha^{\prime}}}{\Gamma\left(2-3 \alpha^{\prime}\right) \Gamma\left(2-\alpha^{\prime}\right)}-\left(\frac{\left(4-3 \alpha^{\prime}\right)}{\Gamma\left(3-3 \alpha^{\prime}\right)}+\frac{\Gamma\left(3-2 \alpha^{\prime}\right)}{\Gamma\left(2-\alpha^{\prime}\right)^{2} \Gamma\left(3-3 \alpha^{\prime}\right)}\right) \frac{x^{2-\alpha^{\prime}}}{\Gamma\left(2-\alpha^{\prime}\right)} \\
& +\frac{x^{1-3 \alpha^{\prime}}}{\Gamma(1+3 \alpha)} t^{3 \alpha}\left(-\left(\frac{\left(2-\alpha^{\prime}\right)\left(3-2 \alpha^{\prime}\right)}{\Gamma\left(4-3 \alpha^{\prime}\right)}+\frac{\Gamma\left(4-2 \alpha^{\prime}\right)}{\Gamma\left(2-\alpha^{\prime}\right)^{2} \Gamma\left(4-3 \alpha^{\prime}\right)}\right) \frac{x^{3-\alpha^{\prime}}}{\Gamma\left(2-\alpha^{\prime}\right)}+\frac{3 \alpha \Gamma\left(3-2 \alpha^{\prime}-\beta\right) x^{2-\alpha^{\prime}-\beta}}{\Gamma\left(2-\alpha^{\prime}\right)^{2} \Gamma\left(1-\alpha^{\prime}\right) \Gamma(3-\beta) \Gamma\left(3-3 \alpha^{\prime}-\beta\right)}-\frac{\alpha^{\prime}\left(1+\alpha^{\prime}\right) \Gamma\left(3-\alpha^{\prime}-\delta\right) x^{2-\delta}}{\Gamma\left(1-\alpha^{\prime}\right) \Gamma(4-\delta) \Gamma\left(3-2 \alpha^{\prime}-\delta\right)}\right. \\
& +\frac{3}{\Gamma\left(2-\alpha^{\prime}\right)}\left(\begin{array}{l}
\frac{x^{1-\beta}}{\Gamma\left(2-2 \alpha^{\prime}-\beta\right)}+\frac{\left(4-3 \alpha^{\prime}\right) x^{2-\beta}}{\Gamma\left(3-2 \alpha^{\prime}-\beta\right)}+\frac{\left(3-2 \alpha^{\prime}\right)\left(2-\alpha^{\prime}\right) x^{3-\beta}}{\Gamma\left(4-2 \alpha^{\prime}-\beta\right)}+\frac{\left(3-2 \alpha^{\prime}\right) x^{2-\beta}}{\Gamma\left(2-\alpha^{\prime}\right)^{2} \Gamma\left(3-2 \alpha^{\prime}-\beta\right)}+\frac{\Gamma\left(4-2 \alpha^{\prime}\right) x^{3-\beta}}{\Gamma\left(2-\alpha^{\prime}\right)^{2} \Gamma\left(4-2 \alpha^{\prime}-\beta\right)} \\
+\frac{3 \alpha \Gamma\left(3-2 \alpha^{\prime}-\beta\right) x^{2-2 \beta}}{\Gamma\left(2-\alpha^{\prime}\right) \Gamma\left(1-\alpha^{\prime}\right) \Gamma(3-\beta) \Gamma\left(3-2 \alpha^{\prime}-2 \beta\right)}-\frac{\alpha^{\prime}\left(1+\alpha^{\prime}\right) \Gamma\left(3-\alpha^{\prime}-\delta\right) x^{2+\alpha^{\prime}-\beta-\delta}}{\Gamma\left(1-\alpha^{\prime}\right) \Gamma(4-\delta) \Gamma\left(3-\alpha^{\prime}-\beta-\delta\right)}-\frac{\alpha \Gamma\left(1+2 \alpha^{\prime}\right) \Gamma\left(3-\alpha^{\prime}-\delta\right) x^{2-\alpha^{\prime}-\beta}}{\Gamma\left(1-\alpha^{\prime}\right) \Gamma(1+\alpha)^{2} \Gamma(3-\beta)} \\
\left.\frac{\Gamma\left(2-\alpha^{\prime}\right)}{\Gamma\left(2-2 \alpha^{\prime}\right)}+\frac{\Gamma\left(3-\alpha^{\prime}\right) x}{\Gamma\left(3-2 \alpha^{\prime}\right)}\right)-\frac{x^{1+\alpha-\delta}}{\Gamma\left(2-2 \alpha^{\prime}-\delta\right)}-\frac{\left(4-3 \alpha^{\prime}\right) x^{2+\alpha^{\prime}-\delta}}{\Gamma\left(3-2 \alpha^{\prime}-\delta\right)}-\frac{\left(3-2 \alpha^{\prime}\right)\left(2-\alpha^{\prime}\right) x^{3+\alpha^{-}-\delta}}{\Gamma\left(4-2 \alpha^{\prime}-\delta\right)}-\frac{\Gamma\left(3-2 \alpha^{\prime}\right) x^{2+\alpha^{-}-\delta}}{\Gamma\left(2-\alpha^{\prime}\right)^{2} \Gamma\left(3-2 \alpha^{\prime}-\delta\right)} \\
\frac{\Gamma\left(4-2 \alpha^{\prime}\right) x^{3+\alpha-\delta}}{\Gamma\left(2-\alpha^{\prime}\right)^{2} \Gamma\left(4-2 \alpha^{\prime}-\delta\right)}-\frac{3 \alpha \Gamma\left(3-2 \alpha^{\prime}-\beta\right) x^{2+\alpha^{\prime}-\beta-\delta}}{\Gamma\left(2-\alpha^{\prime}\right) \Gamma\left(1-\alpha^{\prime}\right) \Gamma(3-\beta) \Gamma\left(3-2 \alpha^{\prime}-\beta-\delta\right)}-\frac{\alpha^{\prime}\left(1+\alpha^{\prime}\right) \Gamma\left(3-\alpha^{\prime}-\delta\right) x^{2+2 \alpha-2 \delta}}{\Gamma\left(1-\alpha^{\prime}\right) \Gamma(4-\delta) \Gamma\left(3-\alpha^{\prime}-2 \delta\right)} \\
\frac{\alpha \Gamma\left(1+2 \alpha^{\prime}\right)\left(1+\alpha^{\prime}\right)(1+x) x^{1+\alpha^{-}-\delta}}{\Gamma\left(1-\alpha^{\prime}\right) \Gamma(1+\alpha)^{2} \Gamma(4-\delta) \Gamma\left(2-\alpha^{\prime}\right)}
\end{array}\right)
\end{aligned}
$$

\section{Application of ADM}

In the light of the ADM, we may rewrite (1) as

$$
L_{t}^{\alpha} u=D_{t}^{\alpha} D_{x}^{\beta} u-D_{x}^{\alpha^{\prime}} u(1+u)+3 D_{x}^{\alpha^{\prime}} u D_{x}^{\beta} u+u D_{x}^{\delta} u
$$

where,

$$
L_{t}^{\alpha}(.)=\frac{1}{\Gamma(1-\alpha)} \int_{0}^{t} \frac{\partial(.)}{\partial t}(t-\tau)^{-\alpha} d \tau
$$

is an easily invertible operator in the Caputo's sense. According to the ADM, (40) can be written as $u(x, t)=u(0)+\left.L_{t}^{\alpha} u\right|_{t=0}+L_{t}^{-\alpha}\left[D_{t}^{\alpha} D_{x}^{\beta} u-D_{x}^{\alpha^{\prime}} u(1+u)+3 D_{x}^{\alpha^{\prime}} u D_{x}^{\beta} u+u D_{x}^{\delta} u\right]$

with

$$
L_{t}^{-\alpha}(.)=\frac{1}{\Gamma(\alpha)} \int_{0}^{t}(.)(t-\tau)^{\alpha-1} d \tau
$$

Applying the given initial condition (28), (42) becomes

$$
u(x, t)=x+L_{t}^{-\alpha}\left[D_{t}^{\alpha} D_{x}^{\beta} u-D_{x}^{\alpha^{\prime}} u(1+u)+3 D_{x}^{\alpha^{\prime}} u D_{x}^{\beta} u+u D_{x}^{\delta} u\right]
$$

In view of the ADM, the solution is expressed in a series form given by 


$$
u(x, t)=\sum_{n=0}^{\infty} u_{n}(x, t)
$$

Substituting (45) into (44), the resulting recurrence relation is established for $n \geq 0$ :

$$
u_{n+1}=L_{t}^{-\alpha}\left[D_{t}^{\alpha} D_{x}^{\beta} u-D_{x}^{\alpha^{\prime}} u(1+u)+3 D_{x}^{\alpha^{\prime}} u D_{x}^{\beta} u+u D_{x}^{\delta} u\right]
$$

where,

$$
u_{0}(x, t)=x
$$

Then for $n=0,1,2$, we also obtain (36)-(39) respectively.

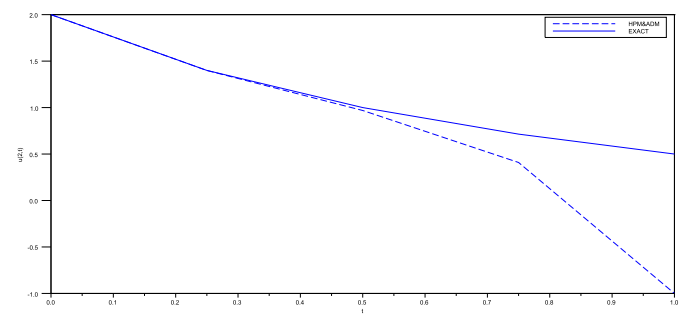

Fig 1. Behavior of $u(x, t)$ for varying $t$, when $x=2$

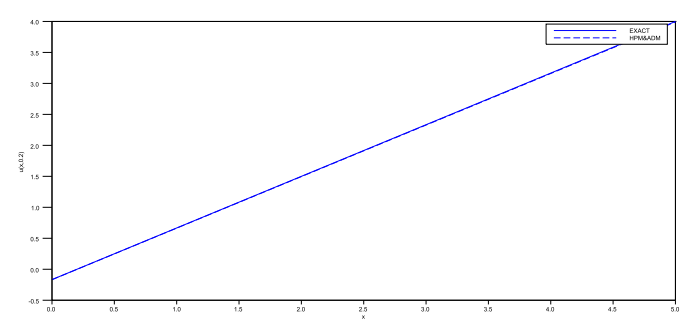

Fig 2. Behavior of $u(x, t)$ for varying $x$, when $t=0.2$.

Table 3.

\begin{tabular}{ccccc}
\hline $\mathbf{t}$ & Exact & HPM\&ADM & Error & Percentage Error \\
\hline 0.0000 & 5.0000 & 5.0000 & 0.0000 & 0.0000 \\
0.1000 & 4.4000 & 4.4000 & 0.0000 & 0.0000 \\
0.2000 & 3.9090 & 3.9090 & 0.0000 & 0.0000 \\
0.3000 & 3.5000 & 3.5000 & 0.0000 & 0.0000 \\
0.4000 & 3.1538 & 3.1218 & 0.0312 & 0.9900 \\
0.5000 & 2.8571 & 2.7440 & 0.1131 & 3.9600 \\
0.6000 & 2.6000 & 2.2840 & 0.3160 & 12.154 \\
0.7000 & 2.3750 & 1.6279 & 0.7471 & 31.453 \\
0.8000 & 2.1765 & 0.6096 & 1.5669 & 71.992 \\
0.9000 & 2.0000 & -1.0000 & 3.0000 & 150.00 \\
\hline
\end{tabular}

\section{Discussion and Results}

First, we observe that though the procedure of obtaining approximate solutions using the HPM and ADM differs, the results obtained are exactly the same.

Now, setting $\delta=3, \beta=2$ and $\alpha^{\prime}=1$, in (1), it was mentioned above that the explicit analytical approximate solution to the resulting $\mathrm{fBP}$ has been obtained. Setting $\delta=3, \beta=2$ and $\alpha^{\prime}=1$, in (36)-(39), we obtain similar result reported in (18) of [8] and expressed as

$$
\begin{gathered}
u(x, t)=x-\frac{1+x}{\Gamma(1+\alpha)} t^{\alpha}+\frac{2(1+x)}{\Gamma(1+2 \alpha)} t^{2 \alpha} \\
-\frac{(1+x)\left[4 \Gamma(1+\alpha)^{2}+\Gamma(1+2 \alpha)\right]}{\Gamma(1+3 \alpha) \Gamma(1+\alpha)^{2}} t^{3 \alpha}
\end{gathered}
$$

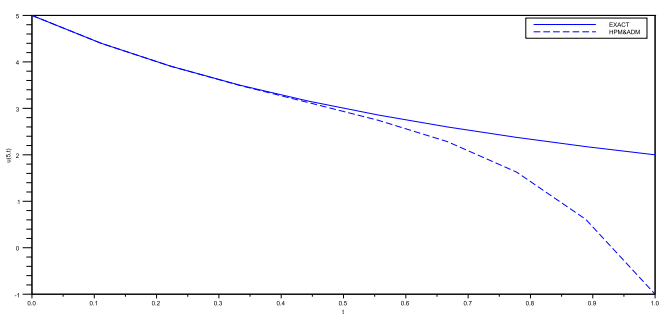

Fig 3. Behavior of $u(x, t)$ for varying $t$, when $x=5$.

Thus, the exact solution to BP equation is obtained when $\alpha=1$ in (48) as

$$
\begin{gathered}
u_{0}(x, t)=x \\
u_{1}(x, t)=-(1+x) t \\
u_{2}(x, t)=(1+x) t^{2} \\
u_{3}(x, t)=-(1+x) t^{3}
\end{gathered}
$$

Then in view of (35) and (46), ${ }^{u_{n}}$ satisfies

$$
u_{n}(x, t)=(-1)^{n}(1+x) t^{n}
$$

In other words, the exact solution is given as

$$
u(x, t)=\lim _{j \rightarrow \infty} \sum_{n=0}^{j} u_{n}(x, t)=\frac{1+x}{1+t}-1
$$

which is just the same as that obtained employing Variational iteration method (VIM) [3] and the classical Lie method [2]. 


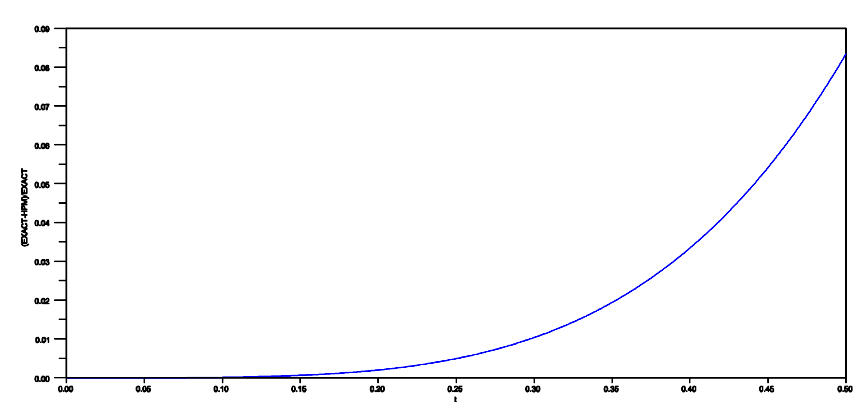

Fig 4. Error difference of the exact solution and the HPM for $x=5$ as $t$ varies.

Now, setting $\alpha=1, x=2$ in (48) and (54), we obtain respectively

$u(2, t)=2-3\left(t-t^{2}+t_{3}-\ldots\right)$

$u(2, t)=\frac{3}{1+t}-1$
Likewise, we may set $\alpha=1, t=0.2$

to respectively obtain for (48) and (54),

$u(x, 0.2)=x-(1+x) 0.2+(1+x) 0.2^{2}-(1+x) 0.2^{3}+\ldots$

u $(x, 0,2)=\frac{1+x}{1+2}-1$

Furthermore, setting $\alpha=1, x=5$ in (48) and (54), we obtain respectively

$u(5, t)=5-6\left(t-t^{2}+t_{3}-\ldots\right)$

$u(5, t)=\frac{5}{1+t}-1$

Figure I presents (55) for $\alpha=1$ and (56), where $x=2, t$ varies, figure II presents (57) for $\alpha=1$ and (58), where $t=0.2, x$ varies, figure III presents (59) for $\alpha=1$ and (60), where $x=5, t$ varies and figures IV and $\mathrm{V}$ presents the error difference between the exact solution and the solution obtained by HPM for $x=5$ and $x=2$ respectively as $\mathrm{t}$ varies.

Tables 1,2 and 3, show the numerical values of the error between the exact result and the fourth-order approximate solution obtained from the HPM/ADM. We can observe the accuracy met for small values of the varying parameter, but as these values increase, inaccuracy takes the same toll. This can be attributed to the few components of our approximate solution in both cases.

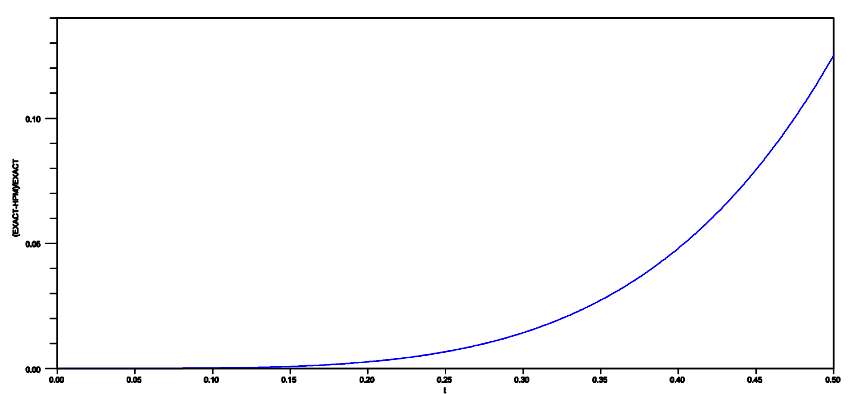

Fig IV. Error difference of the exact solution and the HPM for $x=2$ as $t$ varies.
On the other hand, we can observe from figures IV and $\mathrm{V}$ that the approximate techniques employed in this study agree excellently with the given exact result until at such time when there is need to add more terms to the approximate solution, since we used a four-term approximation in our study. Though, one must note that more terms are required for the solutions obtained through the given approximate techniques in order to keep the error to the barest minimum as time increases, it is imperative to say that the approximate techniques employed proved effective and efficient.

\section{Conclusion}

Through the use of two approximate techniques viz HPM and ADM, exact and explicit approximate solutions to the MFBP, $\mathrm{ABP}$ and BP equations were obtained in this paper. It was found out in this paper that the employed approximate techniques give similar results that agree excellently with the known exact result obtained through the VIM, classical Lie method and analytical methods. Attempts were made at presenting the error difference between the exact result and the obtained approximate solutions, results indicate accuracy, effectiveness and efficiency on the part of the employed approximate techniques. This work represents a progressive step towards developing a complete analysis and gaining full understanding of the effect of approximate techniques to realistic physical models especially those modeled by multi-order fractional differential equations. Future works can extend their scope towards the effect of these approximate techniques on model equations such as the one treated in this paper with external forcing as well as time delay.

\section{References}

[1] K. Fellnerand and C. Schmeiser. Burgers-Poisson: a nonlinear dispersive wave equation: SIAM J. Appl. Math. 64, 1509-1525, 2004.

[2] N. C. Turgay and E. Hizel. Group Invariant Solutions for Burgers-Poisson Equation: Int. Math. Forum, 55, 2701-2710, 2007.

[3] E. Hizel and S. Kucukarslan. A numerical analysis of Burgers-Poisson equation using variational iteration method: $3^{\text {rd }}$ WSEAS ICATM, Spain, December, 14-16, 2007.

[4] R. Fetecau and D. Levy. Approximate model equation for water waves: Comm. Math. Sci. 3, 159-170, 2005.

[5] M. Kato. Large time behavior of solutions to the BurgersPoisson equations: Osaka J. Math. 4(4), 923-943, 2007.

[6] K. Hamer. Nonlinear effects on the propagation of sound waves in a radiating gas: Quart. J. Mech. Appl. Math. 24, $155-168,1971$. 
[7] W. G. Vincent and C. H. Kruger. Introduction to physical gas dynamics, Wiley, New York, 1965.

[8] C. Zeng, Q. Yang and B. Zhang. Homotopy perturbation method for fractional-order Burgers-Poisson equation. Available from arXiv:1003.1828v1 [nlin.PS], 2010.

[9] J. H. He. Homotopy Perturbation Technique: Comput. Meth. Appl. Mech. Eng. 178, 257-262, 1999.

[10] J. H. He. An Elementary introduction to the homotopy perturbation method. Computers and Mathematics with Applications, 57(3), 410-412, 2009.

[11] J. H. He. The homotopy perturbation method for nonlinear oscillators with discontinuities, Appl.Math.Comput.151, 287-292, 2004.

[12] J. H. He. Homotopy Perturbation Method: A new nonlinear analytical technique, Appl. Math. Comput. 137, 73-79, 2003.

[13] J. H. He. Recent development of the homotopy perturbation method, Topols. Meth. Nonlinear Anal. 31, 205-209, 2008.

[14] G. Adomian. Solving Frontier Problems of Physics: The Decomposition Method. Kluwer Academic Publishers, Boston 1994

[15] G. Adomian. Solutions of nonlinear PDE. Applied Mathematical Letters 11(13), 121-123, 1998.

[16] P. Pue-on and N. Viriyapong. Modified Adomian Decomposition Method for Solving Particular Third-Order Ordinary Differential Equations. Applied Mathematical Sciences 6(30), 1463-1469, 2012.

[17] R. Montazeri. A Concrete Application of Adomian Decomposition Method. Int. J. Contemp. Math. Sciences 7(24), 1185-1192, 2012.

[18] H. Fatoorehchi and H. Abolghasemi. Adomian Decomposition Method to Study Mass Transfer from a Horizontal Flat Plate subject to Laminar Fluid Flow. Advances in Natural and Applied Sciences 5(1), 26-33, 2011.

[19] J. I. Nwamba. Delayed Mathieu equation with fractional order damping: An approximate analytical solution. Int. J.
Mech. Appl. 3 (4), 70-76, 2013.doi:105923/j.mechanics 20130304.02.

[20] Arikoglu and I. Ozkol. Solution of fractional integrodifferential equations by using fractional differential transform method: Chaos, Solitons and Fractals, 40, 521$529,2009$.

[21] Ghorbani. Towards a new analytical method for solving fractional differential equations: Comp. Math. Appl. Mech. Eng., 197, 4173-4177, 2008.

[22] M. M. Ghasemi. Numerical solution of the nonlinear integro-differential equations: Wavelet-Galerkin method and Homotopy perturbation method, Appl. Math. Comput., 188, 450-455, 2007.

[23] Taiwo and O. S. Adetunde. Approximation of Multi-order fractional differential equations by an iterative decomposition method: American Journal of Engineering Science and Technology Research, 1(2), 10-18, 2013.

[24] K. Hanan. The Homotopy analysis method for solving Multi-fractional order integro-differential equations: J. AlNahrain Univ., 14(3), 139-143, 2011.

[25] Podlubny. Fractional Differential Equations, Academic press, San Diego, California, 1999.

[26] J. West, M. Bolognab and P. Grigolini. Physics of Fractal operators, Springer, New York, 2003.

[27] H. M. Srivasta, R.K. Saxena. Operators of fractional integration and their applications, Appl. Math. Comput., 118 , $1-52,2001$

[28] R. L. Bagley, P. J. Torvik. A theoretical basis for the application of fractional calculus to viscoelasticity, J. Rheol., 27, 201-210, 1983.

[29] R. Hilfer (ED). Applications of Fractional Calculus in Physics, Applications of Fractional Calculus in Physics, 2000.

[30] Ross. A brief history and exposition of the fundamental theory of fractional calculus, In: Fractional calculus and its applications. Springer lecture notes in mathematics, 57, 1-36, 1975. 